\title{
Modified Huo-Luo-Xiao-Ling Dan Suppresses Adjuvant Arthritis by Inhibiting Chemokines and Matrix-Degrading Enzymes
}

\author{
Siddaraju M. Nanjundaiah, ${ }^{1}$ David Y.-W. Lee, ${ }^{2}$ Zhongze Ma, ${ }^{2}$ Harry H. S. Fong, ${ }^{3}$ \\ Lixing Lao, ${ }^{4}$ Brian M. Berman, ${ }^{4}$ and Kamal D. Moudgil ${ }^{1}$ \\ ${ }^{1}$ Department of Microbiology and Immunology, University of Maryland School of Medicine, HSF-1, Suite 380, \\ 685 West Baltimore Street, Baltimore, MD 21201, USA \\ ${ }^{2}$ Mailman Research Center, McLean Hospital, Harvard Medical School, Belmont, MA 02478, USA \\ ${ }^{3}$ Department of Medicinal Chemistry and Pharmacognosy, University of Illinois at Chicago, Chicago, IL 60612, USA \\ ${ }^{4}$ Center for Integrative Medicine, University of Maryland School of Medicine, East Hall, 520 W. Lombard Street, Baltimore, \\ MD 21201, USA
}

Correspondence should be addressed to Kamal D. Moudgil, kmoud001@umaryland.edu

Received 18 August 2011; Revised 24 October 2011; Accepted 15 November 2011

Academic Editor: Boli Zhang

Copyright ( 2012 Siddaraju M. Nanjundaiah et al. This is an open access article distributed under the Creative Commons Attribution License, which permits unrestricted use, distribution, and reproduction in any medium, provided the original work is properly cited.

\begin{abstract}
Rheumatoid arthritis (RA) is a chronic inflammatory disease affecting the joints that can lead to deformities and disability. The prolonged use of conventionally used drugs is associated with severe adverse reactions. Therefore, safer and less expensive therapeutic products are continually being sought. Huo-Luo-Xiao-Ling dan (HLXL), a traditional Chinese herbal mixture, and its modified versions possess anti-arthritic activity. In this paper, we examined the influence of modified HLXL on two of the key mediators of arthritic inflammation and tissue damage, namely, chemokines and matrix-metalloproteinases (MMPs) in the rat adjuvant-induced arthritis (AA) model of RA. We treated arthritic Lewis rats with HLXL $(2.3 \mathrm{~g} / \mathrm{kg})$ by daily gavage beginning at the onset of AA. The control rats received the vehicle. At the peak phase of AA, rats were sacrificed and their draining lymph node cells (LNC) and spleen adherent cells (SAC) were tested. The HLXL-treated rats showed a significant reduction in the levels of chemokines (RANTES, MCP-1, MIP-1 $\alpha$, and GRO/KC), MMPs (MMP 2 and 9), as well as cytokines (IL-6 and IL-17) that induce them, compared to the control vehicle-treated rats. Thus, HLXL controls arthritis in part by suppressing the mediators of immune pathology, and it might offer a promising alternative/adjunct treatment for RA.
\end{abstract}

\section{Introduction}

Rheumatoid arthritis (RA) is a chronic debilitating autoimmune disease that affects over 1 percent of the population worldwide [1]. The commonly used drugs such as nonsteroidal anti-inflammatory drugs (NSAIDS) and biologics (e.g., antitumor necrosis- $\alpha$ antibody) are effective in alleviating the symptoms of the disease. However, the prolonged use of these drugs is associated with severe adverse reactions $[2,3]$. In addition, these drugs are expensive, and not all patients respond well to them. In view of these limitations, it is essential to continue the search for safer and less expensive alternatives to the conventionally used drugs $[4,5]$. Natural plant products represent a promising group of therapeutic agents for arthritis. However, one of the major concerns in seriously considering these products for therapeutic purposes is that the mechanisms of action of many of them are poorly defined, if at all.

RA primarily targets the joints, and is characterized by inflammatory synovitis mediated by leukocytes and the proinflammatory cytokines secreted by them $[1,6]$. The migration of leukocytes from the peripheral blood into the joints is directed by chemotactic cytokines (chemokines) [7]. Furthermore, severe arthritis is associated with cartilage and bone damage, which is mediated in part by the matrixdegrading enzymes, matrix metalloproteinases (MMPs) [8, 9]. Therefore, chemokines and MMPs are attractive targets for the treatment of arthritis $[10,11]$. 
Chemokines are small, biologically active molecules that attract specific populations of inflammatory cells and regulate their trafficking to the site of inflammation. Among the chemokines that play an important role in inflammation, including RA, are regulated upon activation, normal $\mathrm{T}$ cell expressed, and secreted (RANTES), also known as chemokine $\mathrm{C}-\mathrm{C}$ motif ligand 5 (CCL5); monocyte chemotactic protein-1 (MCP-1), or CCL2; macrophage inflammatory protein-1 $\alpha$ (MIP-1 $\alpha$ ), or CCL3; and growth regulated oncogene-keratinocyte chemoattractant $(\mathrm{GRO} / \mathrm{KC})$, or chemokine C-X-C motif ligand 1 (CXCL1) [12-15]. The chemokines interact with their receptors which belong to seventransmembrane G-protein-coupled molecules. Henceforth, for simplicity we will refer to the 4 chemokines tested in this study by their common names (RANTES, MCP- 1 , MIP- $1 \alpha$, and $\mathrm{GRO} / \mathrm{KC}$ ).

MMPs are a group of zinc-dependent endopeptidases that can degrade the extracellular matrix (ECM). Among the MMPs, MMP-2 (gelatinase A) and MMP-9 (gelatinase B) are especially important in collagen degradation [16]. The expression/activity of MMPs can be modulated by the proinflammatory cytokines interlukin-6 (IL-6) and IL-17 that are expressed in the synovial tissue and synovial fluid in the joints of RA patients $[8,17]$.

Huo-luo-xiao-ling dan (HLXL), a traditional Chinese herbal formula, and its modified versions have been used in folk medicine over centuries to treat inflammatory arthritis or joint pain, referred to as the "Bi syndrome" $[18,19]$. The use of a combination of multiple herbs is designed to exploit the additive or synergistic activities of individual herbs, as well as to balance or neutralize the toxic effects of certain herbal components by others in the mixture [20]. In our previous studies in animal models of arthritis, we have shown that oral administration of modified HLXL, a well-characterized herbal mixture [21-23], to rats can attenuate inflammatory paw edema as well as the swelling and pain associated with clinical arthritis. In addition, HLXL modulates the balance of pro- versus anti-inflammatory cytokines [24]. In this study, we examined the effect of HLXL on specific chemokines and MMPs, which play an important role in the pathogenesis of AA [25]. As the expression/activity of certain chemokines as well as MMPs is influenced significantly by the proinflammatory cytokines (IL-17 and IL-6), we also tested the effect of HLXL on these two cytokines. We observed that HLXL treatment of arthritic rats attenuates the progression of AA by inhibiting chemokines (RANTES, MCP-1, MIP- $1 \alpha$, and GRO/KC), MMPs (MMP-2 and MMP-9), and cytokines (IL-6 and IL-17). To the best of our knowledge, this is the first report describing these HLXL-induced changes in arthritis.

\section{Materials and Methods}

2.1. Animals. Five- to six-week-old male Lewis (LEW/Hsd) (RT.1 $\left.1^{1}\right)$ rats were purchased from Harlan Sprague-Dawley (HSD) (Indianapolis, IN, USA) and then maintained in the animal care facility of the University of Maryland School of Medicine, Baltimore. All experimental procedures performed on these rats were in accordance with the guidelines of the Institutional Animal Care and Use Committee (IACUC).

2.2. Composition and Characteristics of HLXL. The traditional Huo-Luo-Xiao-Ling Dan (HLXL) consists of four herbs, namely, Danggui (Angelica sinensis (Oliv.) Diels), Danshen (Salvia miltiorrhiza Bge.), Ruxiang (Boswellia carterii Birdw.), and Moyao (Commiphora myrrha Engl.). We have previously reported [21-23] the rationale and the nature of modification of this original formula, and the modified HLXL is a defined mixture of 11 herbs, namely, Ruxiang (Boswellia carterii Birdw.), Qianghuo (Notopterygium incisum Ting ex H.T. Chang), Danggui (Angelica sinensis (Oliv.) Diels), Baishao (Paeonia lactiflora Pall.), Gancao (Glycyrrhiza uralensis Fisch.), Yanhusuo (Corydalis yanhusuo W.T. Wang.), Danshen (Salvia miltiorrhiza Bge.), Chuanxiong (Ligusticum chuanxiong S.H. Qiu.), Qin jiao (Gentiana macrophylla Pall.), Guizhi (Cinnamomum cassia Presl.), and Duhuo (Angelica pubescens Maxim). The compounds isolated from HLXL include steroids, terpenes, alkaloids, flavonoids, glycosides, and acids [21]. The methods for the preparation of HLXL, for the characteristics of its component herbs, and for the assessment of its toxicity have been described in detail elsewhere [22, 23]. As in our earlier studies $[23,24]$ the batch of HLXL tested in this study was thoroughly characterized by HPLC fingerprinting, which were characterized by the peak shapes, numbers, intensities, and retention times of all individual compounds (data not shown). Moreover, the marker compounds, swertiamarin (from Gentiana macrophylla), osthole (Angelica pubescens), paeoniflorin (Paeonia lactiflora), iso-imperatorin (Notopterygium incisum), liquiritin (Glycyrrhiza uralensis), columbianadin (Angelica pubescens), liquiritigenin (Glycyrrhiza uralensis), falcarindiol (Notopterygium incisum), angelol B (Angelica pubescens), cryptotanshinone (Salvia miltiorrhiza), bergapten (Angelica pubescens), tanshinone IIA (Salvia miltiorrhiza), senkyunolide A (Ligusticum chuanxiong), ostruthin (Notopterygium incisum), phenethyl transferulate ( $N$. incisum), and anhydronotoptol ( $N$. incisum), served as references for quality control purposes. In a recent study [21], we reported that some of the above-mentioned herbs other than the 4 traditionally used in classical HLXL, possessed compounds that served as ligands for the enzyme cyclo-oxygenase 2 (COX-2). This further reinforces in part the rationale for the modification of the original HLXL formula.

2.3. Treatment of Arthritic Rats with HLXL. Lewis rats were immunized subcutaneously (s.c.) with $1 \mathrm{mg} /$ rat heat-killed M. tuberculosis H37Ra (Mtb) (Difco, Detroit, MI) in $200 \mu \mathrm{L}$ of mineral oil (Sigma-Aldrich) at the base of the tail. Following the onset of arthritis, these rats were randomly divided into two groups (experimental and control). HLXL was finely powdered and suspended in $200 \mathrm{~mL}$ of water using a pestle and mortar, and it was fed $(2.3 \mathrm{~g} / \mathrm{kg})$ to the experimental group of rats using a gavage needle (FNC-16-3, Kant Scientific Corporation, Torrington, CT, US) beginning on the day of onset of arthritis (d 10) and then continued 
up to the peak phase of AA (d 18). On the corresponding days, the control group of rats received water (the vehicle) by gavage. All rats were examined and graded regularly for the severity of arthritis as described earlier [24, 26]. The test samples were collected from rats when the disease reached the peak phase (d 18) in controls.

\subsection{Preparation and Antigenic Restimulation of Lymph Node} Cells (LNC). Rats treated either with HLXL or with vehicle (water) only were euthanized at the peak phase of AA (d 18) and their draining lymph nodes (para-aortic, inguinal and popliteal) were harvested [26]. A single cell suspension of LNC was prepared in HL-1 serum free medium (Ventrex Laboratories, Portland, ME) supplemented with $2 \mathrm{mM} \mathrm{L-}$ glutamine, $100 \mathrm{U} / \mathrm{mL}$ penicillin $\mathrm{G}$ sodium, and $100 \mu \mathrm{g} / \mathrm{mL}$ streptomycin sulfate. In each experiment, LNC from 3 rats were pooled for testing. These LNC were cultured (8 $\times 10^{6}$ cells/well) in a 6-well plate (Corning Incorporated Corning, NY) for $24 \mathrm{~h}$ at $37^{\circ} \mathrm{C}$ in an atmosphere of $95 \%$ air and $5 \% \mathrm{CO}_{2}$. Sonicated Mtb $(10 \mu \mathrm{g} / \mathrm{mL})$ was used for restimulation of LNC. Keyhole limpet hemocyanin (KLH) was used as the control antigen. Total RNA was prepared from these LNC for testing by quantitative real-time polymerase chain reaction (qRT-PCR) as described below. Alternatively, the LNC culture supernatant was harvested for testing by a Multiplex assay as elaborated below.

2.5. Spleen Adherent Cells (SAC) and Their Stimulation with Sonicated $M t b$. Spleens were harvested from experimen$\mathrm{tal} /$ control rats ( $n=3$ each) at the peak phase of AA (d 18) and a single cell suspension was prepared as described above for LNC. These spleen cells were allowed to settle in a 6-well plate at $37^{\circ} \mathrm{C}$ in RPMI medium supplemented with $5 \%$ fetal bovine serum (FBS), $2 \mathrm{mM} \mathrm{L}$ glutamine, $100 \mathrm{U} / \mathrm{mL}$ penicillin G sodium, and $100 \mu \mathrm{g} / \mathrm{mL}$ streptomycin sulfate. After $90 \mathrm{~min}$, nonadherent cells were removed by washing with HBSS (Invitrogen, Frederick, MD), yielding the SAC [27]. These SAC $\left(1.5-2.0 \times 10^{6}\right.$ cells per well) were restimulated for $6 \mathrm{~h}$ with sonicated $\mathrm{Mtb}(10 \mu \mathrm{g} / \mathrm{mL})$. KLH served as the control antigen. Thereafter, culture supernatants were collected for testing in Multiplex assay. Alternatively, total RNA was prepared from these SAC as described below.

2.6. Determination of Matrix Metalloproteinase (MMP)/ Cytokine mRNA Expression by qRT-PCR. RNA was isolated from LNC and SAC using TRIzol reagent (Invitrogen, Carlsbad, CA) and then cDNA was prepared from RNA using iScript cDNA synthesis kit (Bio-Rad laboratories, Hercules, $\mathrm{CA})$. The resultant cDNA was amplified in an ABI Prism 7900HT cycler (Applied Biosystems) by qRT-PCR using SYBR Green PCR Master Mix (Applied Biosystems) and the appropriate primers for MMP-2, MMP-9, IL-6, and IL-17. These primers were synthesized at the Biopolymer Core Facility, University of Maryland, Baltimore. The mRNA levels of the genes of interest were normalized to the hypoxanthine-guanine phosphoribosyltransferase (HPRT) gene, and the relative gene expression levels were expressed as "fold increase" [26].
2.7. Determination of Chemokine/Cytokine Protein Expression by Multiplex Suspension Bead Array Immunoassay. Multiplex assays were performed at the Cytokine Core Facility (University of Maryland, Baltimore) using the Luminex 100 analyzer (Luminex Corp., Austin, TX) and the level of cytokines (as protein) in $24 \mathrm{~h}$ culture supernatant of LNC and SAC were measured. The Rat chemokine 4-plex kit (Millipore) was used to measure RANTES, MCP-1, MIP- $1 \alpha$, and GRO/KC and the Rat cytokine 2-plex kit was used to measure IL- 6 and IL-17.

2.8. Measurement of MMP Activity Using a Zymogram Assay. The MMP activity in the culture supernatants of LNC and SAC stimulated with or without Mtb was determined using a zymogram assay as described previously [26, 28]. Briefly, culture supernatant was loaded onto a gelatin-coated, precasted polyacrylamide gel (Bio-Rad). Electrophoresis was carried out under SDS-nonreducing conditions at constant voltage. The gel was incubated with $2.5 \%$ Triton X-100 at room temperature for $1-2 \mathrm{~h}$ to remove SDS. The gel was then washed 3-4 times with water to remove Triton $\mathrm{X}-100$ and incubated overnight at $37^{\circ} \mathrm{C}$ in a developing buffer (Tris- $\mathrm{HCl}, \mathrm{pH}$ 7.4) containing $5 \mathrm{mM} \mathrm{CaCl}_{2}, 0.2 \mathrm{M}$ $\mathrm{NaCl}$, and $0.02 \%$ Brij 35. Thereafter, the gel was stained with Coomassie Brilliant Blue R-250. Standard MMP-2 and MMP-9 (Sigma) were used as positive controls. The MMP activity was visualized and scanned after destaining. Thereafter, the intensity of the bands was quantitated by densitometry using Image J software.

2.9. Statistical Analysis. The data were expressed as mean \pm SEM. Student's $t$-test and ANOVA were used to assess the significance of differences using GraphPad Prism version 4.0. A $P$ value of $<0.05$ was considered significant.

\section{Results}

As reported earlier [24], we observed in this study that HLXL treatment of arthritic Lewis rats reduced the severity of AA. The mean arthritic score on d 18 (peak phase of AA), was 2.2 for HLXL-treated group compared to 4.6 for the control water-treated group, and this difference was statistically significant $(P<0.02)$. We then determined the effect of HLXL on specific chemokines, MMPs and cytokines on d 18 of arthritis and compared the results with those obtained from the control rats. The results are presented below.

\subsection{HLXL Treatment Downmodulates Chemokine Production in Arthritic Lewis Rats. Chemokines and their receptors coordinate the movement of cells of the immune system and direct these cells to the site of inflammation. The antigen- draining lymph nodes are the site of initial cellular activation and interactions. In this context, we tested the effect of HLXL treatment on chemokines produced by the draining LNC. Specifically, we tested for RANTES, MCP-1, MIP- $1 \alpha$, and $\mathrm{GRO} / \mathrm{KC}$. These chemokines were measured in culture supernatants of the draining LNC restimulated with sonicated Mtb for $24 \mathrm{~h}$ (Figure 1). We observed a 4.4 -fold}




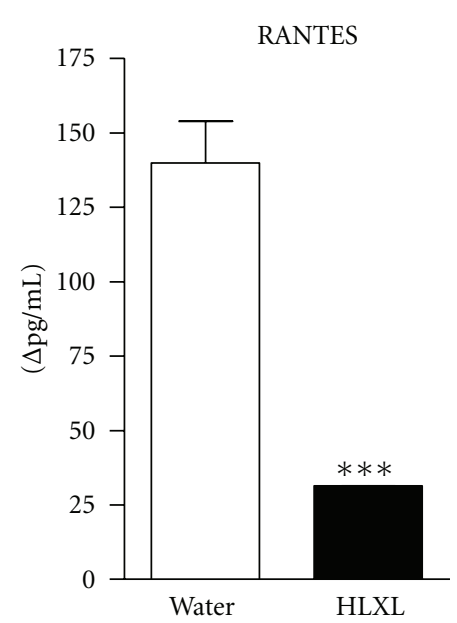

(a)

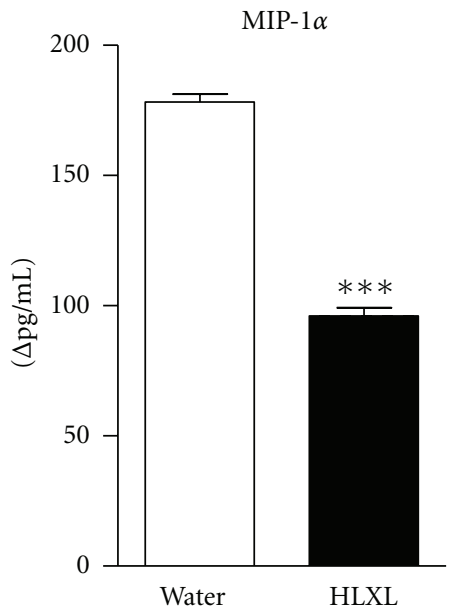

(c)

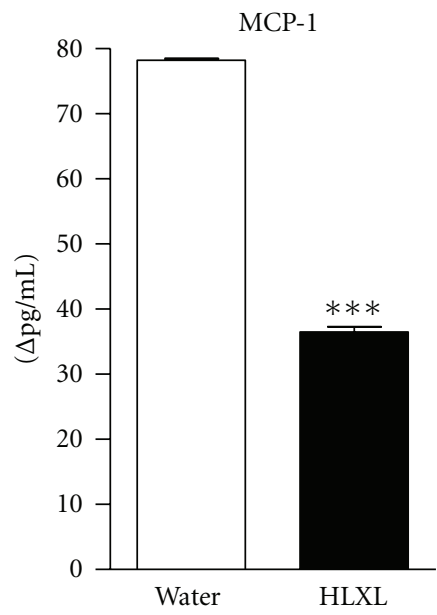

(b)

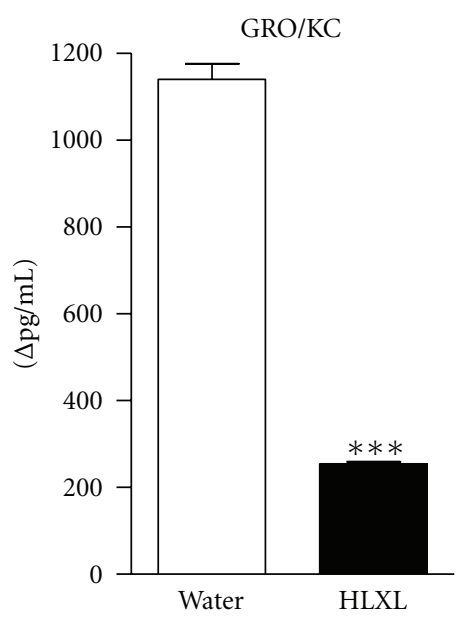

(d)

FIGURE 1: Suppression of chemokines in HLXL-treated arthritic rats. Lymph node cells (LNC) harvested at the peak phase of the disease (d 18) from arthritic rats fed with HLXL or the vehicle (water) were cultured in vitro for $24 \mathrm{~h}$ in the presence or absence of sonicated Mtb. Thereafter, the culture supernatants were harvested and tested for chemokines: RANTES (a), MCP-1 (b), MIP-1 $\alpha$ (c), and GRO/KC (d). The results were expressed as $\Delta \mathrm{pg} / \mathrm{mL}$. Representative results of one of two independent experiments are shown $(* * * P<0.005)(\mathrm{Mtb}=$ heat-killed M. tuberculosis $\mathrm{H} 37 \mathrm{Ra}$ ).

decrease in RANTES in HLXL-treated group, which was highly significant $(P<0.001)$ when compared to the control group. There was a 2.1- and 1.6-fold decrease in MCP-1 $(P<$ $0.00001)$ and MIP- $1 \alpha(P<0.001)$, respectively after HLXL treatment. GRO/KC showed a marked downregulation in HLXL-treated group with a 4.5 -fold decrease $(P<0.001)$ compared to the control group.

\subsection{HLXL Suppresses MMP-9 and MMP-2 Activity in Arthritic} Rats. MMPs mediate the degradation of extracellular matrix macromolecules, and they play an important role in cartilage and bone damage in arthritic joints. Therefore, to further understand the mechanisms underlying the arthritisprotective effect of HLXL, we evaluated the mRNA expression and enzyme activity of MMPs in SAC that were harvested from HLXL-treated or control rats and then restimulated with sonicated Mtb for $24 \mathrm{~h}$. Treatment with HLXL caused a 1.7-fold decrease in the expression of MMP-9 but no significant change in MMP-2 mRNA expression (see Supplementary Figure 1 in Supplementary material available online at doi: 10.1155/2012/589256). Furthermore, there was 2.3-fold suppression in the activity of MMP-9 and a 2.7fold decrease in MMP-2 activity in HLXL-treated group compared to the controls (Figure 2).

3.3. HLXL Treatment Inhibits Antigen-Induced Pro-Inflammatory Cytokine Response in Arthritic Rats. IL-6 and IL-17 are proinflammatory cytokines that have a significant effect on different chemokines and MMPs involved in the arthritogenic processes. Therefore, we tested the levels of these two cytokines (as proteins) in the draining LNC that were harvested from HLXL-treated and control arthritic rats and then restimulated in vitro for $24 \mathrm{~h}$ with sonicated $\mathrm{Mtb}$. There was a significant decrease in the level of IL-6 $(P<0.0002)$ and IL$17(P<0.0001)$ in HLXL-treated rats compared with watertreated rats (Figures 3(a) and 3(b)). HLXL-treated group 


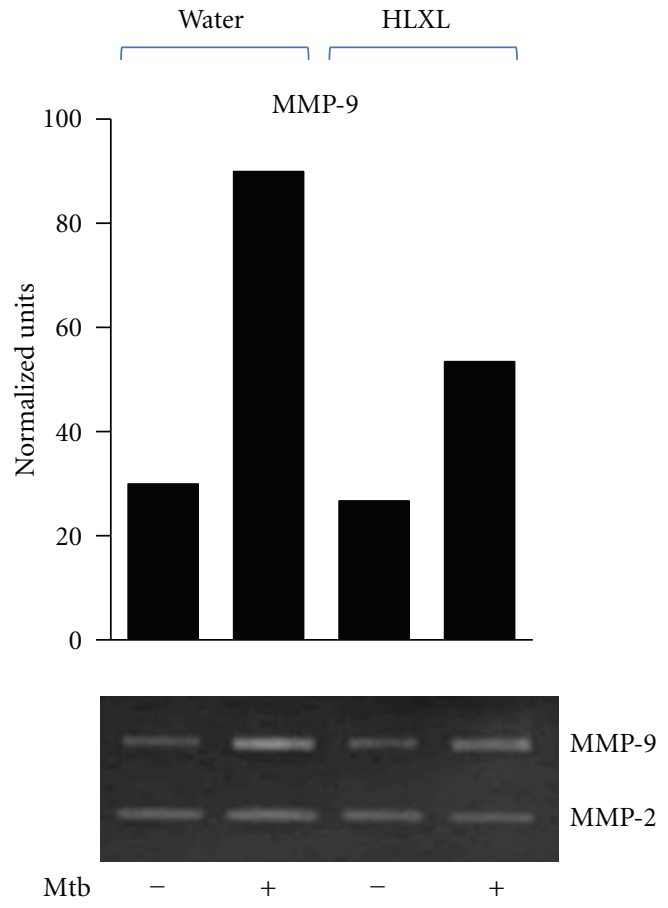

(a)

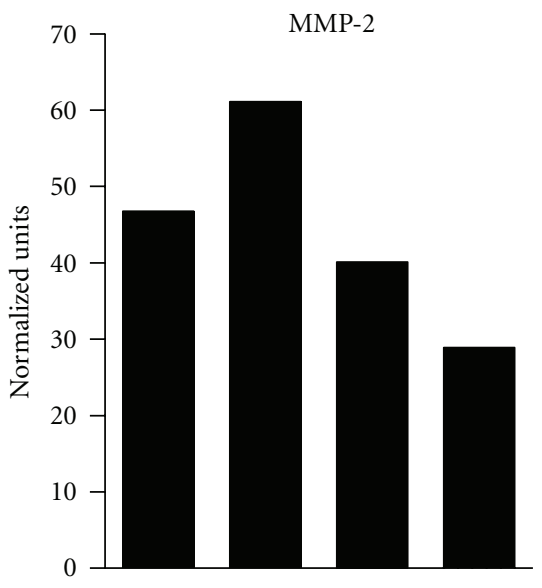

(b)

FIgure 2: The effect of HLXL on MMP activity in arthritic rats. Spleen adherent cells (SACs) harvested from rats fed with HLXL or the vehicle (water) were stimulated for $24 \mathrm{~h}$ with sonicated Mtb. The supernatants obtained from these cultured cells were analyzed for MMP-9 (a) and MMP-2 (b) activity using a zymogram assay. The results are representative of two independent experiments (Mtb = heat-killed $M$. tuberculosis $\mathrm{H} 37 \mathrm{Ra}$ ).

showed a 1.8 -fold decrease in IL-6 and a 4.3-fold de-crease in IL-17 compared to the controls. Furthermore, in SACs stimulated with sonicated Mtb, there was a 14 fold decrease in IL- 6 and a 3.4 fold reduction in IL-17 in HLXL-treated rats compared to vehicle-treated rats (see Supplementary Figure 2). Thus, HLXL inhibited cytokine production in LNC as well as SAC, indicating a systemic effect.

\section{Discussion}

In this study based on the rat AA model of human RA, we observed that HLXL-treated rats had significantly reduced levels of chemokines (RANTES, MCP-1, MIP- $1 \alpha$, and $\mathrm{GRO} / \mathrm{KC}$ ) as well as matrix-degrading enzymes, MMP2 and MMP9. Also reduced were the levels of the proinflammatory cytokines IL-6 and IL-17.

4.1. The Involvement of Chemokines in the Pathogenesis of Arthritis and the Therapeutic Targeting of Chemokines. High levels of RANTES are found in the synovial fluid and serum obtained from patients with RA, and it promotes the migration of mononuclear cells (including lymphocytes) from blood vessels and synovial fluid into the synovium [12]. MCP-1 is chemotactic for monocytes and T lymphocytes [15] whereas GRO- $\alpha$ is a chemoattractant for neutrophils in RA [13]. Elevated levels of MIP- $1 \alpha$ are found in sera and synovial fluid of RA patients suggesting that MIP- $1 \alpha$ plays an important role in the progression of the disease [14]. Initial clinical trials on chemokine blockade in patients with RA suggest that targeting the chemokine and chemokine receptor family might provide a promising therapeutic approach for this deliberating disease [10]. In this context, the results of our study showing that the above-mentioned 4 chemokines are suppressed in HLXL-treated rats compared to the controls are of high significance.

In the AA model, high levels of RANTES are found in the peripheral blood at the onset of the disease, whereas MCP1 is first detected in the synovial tissue and later detected in the peripheral blood on day 18 (peak phase of AA), when joint inflammation is already very active [25]. These results suggest that RANTES is essential for the initiation of the disease, whereas MCP-1 follows the disease onset. Blocking the receptor for MCP-1 before the onset of arthritis in AA affords protection against the disease [29]. In our study reported here, we observed a positive correlation between arthritic scores and MCP-1 levels, with both being reduced after HLXL treatment. Furthermore, our findings of suppression of MCP-1 and RANTES after HLXL treatment are supported by those of another study showing reduced serum levels of MCP-1 and RANTES in RA patients following treatment with formulations of dried encapsulated juice concentrate [30]. Similarly, these chemokines can be suppressed by green tea extract [31]. In regard to $\mathrm{GRO} / \mathrm{KC}$, it was shown in the CIA model that this chemokine is elevated in the synovial 


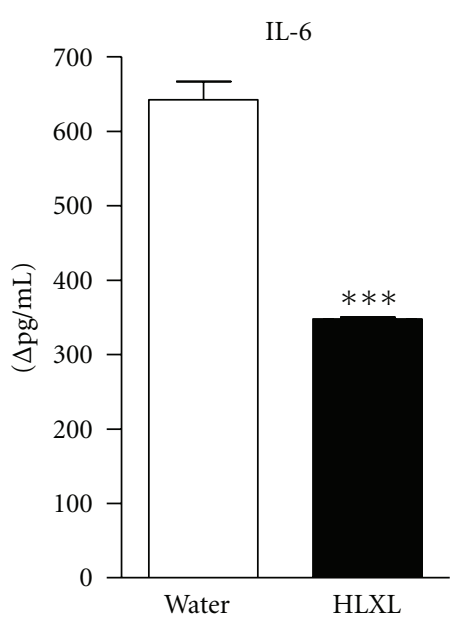

(a)

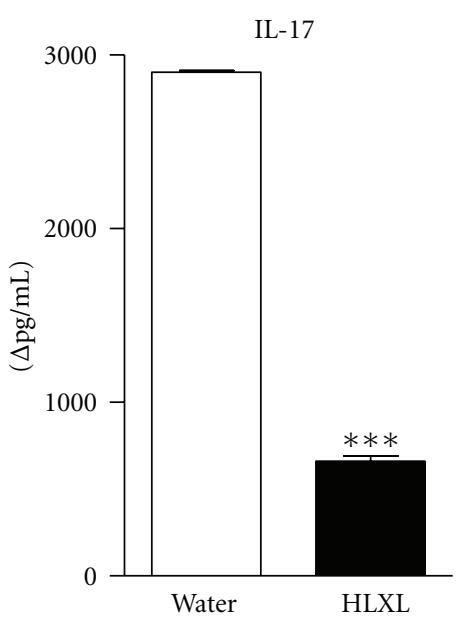

(b)

FIGURE 3: Inhibition of proinflammatory cytokines in HLXL-treated arthritic rats. Lymph node cells (LNCs) harvested on d 18 (peak phase of arthritis) from HLXL-fed or water-fed rats were cultured for $24 \mathrm{~h}$ with or without sonicated Mtb. The culture supernatants were tested for IL-6 (a) and IL-17 (b) using a multiplex assay. The results were expressed as $\Delta \mathrm{pg} / \mathrm{mL}$. The results are representative of two independent experiments. (Mtb = heat-killed M. tuberculosis $\mathrm{H} 37 \mathrm{Ra})$.

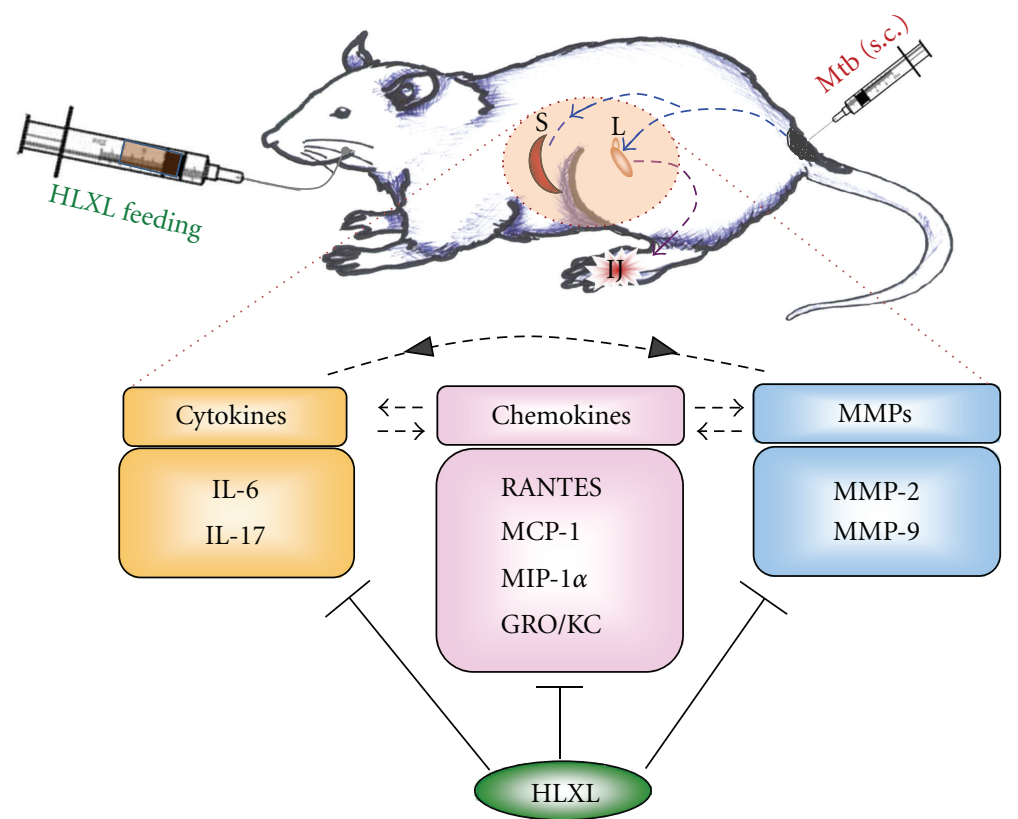

FIGURE 4: A schematic representation of the diverse mechanisms involved in HLXL-induced suppression of AA. HLXL attenuated arthritis via suppressing the disease-regulating chemokines (regulated upon activation, normal T cell expressed and secreted: RANTES; monocyte chemotactic protein-1: MCP-1; macrophage inflammatory protein-1 $\alpha$ : MIP- $1 \alpha$; growth regulated oncogene-keratinocyte chemoattractant: GRO/KC), matrix metalloproteinases (MMP-2 and MMP-9), and cytokines (IL-6 and IL-17). (S; spleen, L; lymph node, IJ; inflamed joint, Mtb; heat-killed Mycobacterium tuberculosis H37Ra).

fluid of arthritic rats and that its levels are reduced following suppression of arthritis by an inhibitor of spleen tyrosine kinase [32]. The results of our study in the AA model showed significant suppression of GRO/KC in HLXL-treated rats, which was associated with reduced severity of AA compared to the control rats.

4.2. The Induction and Regulation of MMPs by Pro-Inflammatory Cytokines and Chemokines, and the Control of MMP
Activity for Therapeutic Purposes. Macrophages and fibroblasts synthesize and secrete several MMPs that participate in the degradation of ECM components and contribute significantly to the tissue damage in RA [9]. Furthermore, MMP-9 is believed to play a key role in directing the migration of macrophages and neutrophils in RA $[8,33]$. Synovial fluid from RA patients contains high levels of MMP-2 and MMP-9 [16]. Therefore, MMP-2 and MMP-9 are attractive targets for the treatment of RA [11]. In this context, our results showing 
that HLXL decreases MMP-9 and MMP-2 activity in AA are of direct relevance to RA.

Besides MMPs, the increased activity of the proinflammatory cytokines such as IL-6 and IL-17 is closely associated with the destruction of cartilage and bone in RA. IL-6 stimulates the production of MMP-2 and MMP-9 [17]. IL-17 also enhances MMP-2 and MMP-9 expression and is synergistic with IL- $1 \beta$ and TNF- $\alpha$ in inducing the expression of other proinflammatory cytokines and MMPs [8]. We observed that both IL-6 and IL-17 (as proteins) were significantly reduced after HLXL treatment. This is the first report on HLXL-mediated suppression of IL-6 in AA. In addition, our study revealed the inhibitory effect of HLXL on IL-17 at the protein level, validating our earlier finding on the reduction of IL-17 mRNA expression [24]. We suggest that the downregulation of IL-6 and IL-17 had a significant influence, either directly or indirectly, on the MMP activity, which was found to be decreased in HLXL-treated rats.

Chemokines also regulate the expression and activity of MMPs. For example, RANTES and MCP-1 activate monocytes to secrete TNF- $\alpha$, and TNF- $\alpha$ in turn stimulates MCP-1, which then induces MMP-9 production by monocytes [34]. On the basis of our results, we suggest that HLXL-induced reduction in MCP-1 might have contributed in part to the reduced production of MMP-9.

\subsection{The Bidirectional Interplay between Pro-Inflammatory} Cytokines and Chemokines. Overproduction of IL- 6 is a characteristic feature of RA, and inhibiting IL-6 activity is one of the therapeutic options for treating RA. Other investigators [35] have shown that RANTES and MCP-1 regulate IL6 production by fibroblast-like synoviocytes in RA. These observations support our results of HLXL-induced concurrent decrease in the above-referenced chemokines as well as IL-6. The other prominent proinflammatory cytokine, IL17 , stimulates the production of multiple chemokines such as MCP-1 and GRO/KC [36]. In our study, we have shown the concurrent downregulation of chemokines and IL-17 in HLXL-treated rats compared to the control rats.

In conclusion, HLXL acts on different chemokines, MMPs, and cytokines and suppresses their expression, which in turn leads to attenuation of inflammatory arthritis (Figure 4). The precise mechanisms of inhibition of these mediators of inflammation are currently under investigation.

\section{Acknowledgments}

This study was supported by PO1 AT002605-05 and R01AT004321 grants from the National Center for Complementary and Alternative Medicine (NCCAM) at the National Institutes of Health, Bethesda, MD. The authors thank Professor Chen Shi-lin, (IMPLAD, Chinese Academy of Medical Sciences, Beijing, China) for assistance in the acquisition and authentication of herbal source materials, Lisa Hester for her help in Multiplex assay, and Rajesh Rajaiah, Shivaprasad H. Venkatesha, Hua Yu, Brian Astry and Ruixin Zhang (University of Maryland) for helpful discussions.

\section{References}

[1] P. E. Lipsky, "Rheumatoid arthritis," in Harrison's Principles of Internal Medicine, A. Fauci, E. Braunwald, D. Kasper et al., Eds., pp. 2083-2092, McGraw Hil, New York, NY, USA, 17th edition, 2008.

[2] G. A. FitzGerald, "Coxibs and cardiovascular disease," The New England Journal of Medicine, vol. 351, no. 17, pp. 1709$1711,2004$.

[3] J. M. Scheiman, "The impact of nonsteroidal anti-inflammatory drug-induced gastropathy," The American Journal of Managed Care, vol. 7, no. 1, supplement, pp. S10-S14, 2001.

[4] D. M. Taibi and C. Bourguignon, "The role of complementary and alternative therapies in managing rheumatoid arthritis," Family \& Community Health, vol. 26, no. 1, pp. 41-52, 2003.

[5] S. H. Venkatesha, B. M. Berman, and K. D. Moudgil, "Herbal medicinal products target defined biochemical and molecular mediators of inflammatory autoimmune arthritis," Bioorganic $\&$ Medicinal Chemistry, vol. 19, no. 1, pp. 21-29, 2011.

[6] P. P. Tak and B. Bresnihan, "The pathogenesis and prevention of joint damage in rheumatoid arthritis: advances from synovial biopsy and tissue analysis," Arthritis \& Rheumatism, vol. 43, no. 12, pp. 2619-2633, 2000.

[7] P. Loetscher and B. Moser, "Homing chemokines in rheumatoid arthritis," Arthritis Research, vol. 4, no. 4, pp. 233-236, 2002.

[8] D. V. Jovanovic, J. Martel-Pelletier, J. A. Di Battista et al., "Stimulation of 92-kd gelatinase (matrix metalloproteinase 9) production by interleukin-17 in human monocyte/macrophages: a possible role in rheumatoid arthritis," Arthritis \& Rheumatism, vol. 43, no. 5, pp. 1134-1144, 2000.

[9] P. S. Burrage, K. S. Mix, and C. E. Brinckerhoff, "Matrix metalloproteinases: role in arthritis," Frontiers in Bioscience, vol. 11, no. 1, pp. 529-543, 2006.

[10] J. J. Haringman, R. L. Oostendorp, and P. P. Tak, “Targeting cellular adhesion molecules, chemokines and chemokine receptors in rheumatoid arthritis," Expert Opinion on Emerging Drugs, vol. 10, no. 2, pp. 299-310, 2005.

[11] C. Jackson, M. Nguyen, J. Arkell, and P. Sambrook, "Selective matrix metalloproteinase (MMP) inhibition in rheumatoid arthritis-targetting gelatinase A activation," Inflammation Research, vol. 50, no. 4, pp. 183-186, 2001.

[12] L. Boiardi, P. Macchioni, R. Meliconi, L. Pulsatelli, A. Facchini, and C. Salvarani, "Relationship between serum RANTES levels and radiological progression in rheumatoid arthritis patients treated with methotrexate," Clinical and Experimental Rheumatology, vol. 17, no. 4, pp. 419-425, 1999.

[13] A. E. Koch, S. L. Kunkel, M. R. Shah et al., "Growth-related gene product $\alpha$. A chemotactic cytokine for neutrophils in rheumatoid arthritis," The Journal of Immunology, vol. 155, no. 7, pp. 3660-3666, 1995.

[14] Y. Hatano, T. Kasama, H. Iwabuchi et al., "Macrophage inflammatory protein 1 alpha expression by synovial fluid neutrophils in rheumatoid arthritis," Annals of the Rheumatic Diseases, vol. 58, no. 5, pp. 297-302, 1999.

[15] S. Shahrara, S. R. Pickens, A. M. Mandelin et al., "IL-17-mediated monocyte migration occurs partially through CC chemokine ligand 2/monocyte chemoattractant protein-1 induction," The Journal of Immunology, vol. 184, no. 8, pp. 44794487, 2010.

[16] Y. Yoshihara, H. Nakamura, K. Obata et al., "Matrix metalloproteinases and tissue inhibitors of metalloproteinases in 
synovial fluids from patients with rheumatoid arthritis or osteoarthritis," Annals of the Rheumatic Diseases, vol. 59, no. 6, pp. 455-461, 2000.

[17] A. E. Kossakowska, D. R. Edwards, C. Prusinkiewicz et al., "Interleukin-6 regulation of matrix metalloproteinase (MMP-2 and MMP-9) and tissue inhibitor of metalloproteinase (TIMP1) expression in malignant non-Hodgkin's lymphomas," Blood, vol. 94, no. 6, pp. 2080-2089, 1999.

[18] B. G. Wan and B. H. Tao, "An 87 cases' clinical observation, arthritis treated by modified Huo-Luo-Xiao-Ling Dan," Jiangxi Journal of Traditional Chinese Medicine, vol. 33, article 12, 2002.

[19] Y. C. Wang, S. Y. Ji, and R. Wang, "The clinical application of Huo-Luo-Xiao-Ling Dan," Jiangxi Journal of Traditional Chinese Medicine, vol. 30, article 53, 2002.

[20] D. Bernsky and R. Barolet, Chinese Herbal Medicine: Formulas and Strategies, Eastland, Seattle, Wash, USA, 1990.

[21] H. Cao, R. Yu, Y. Choi et al., "Discovery of cyclooxygenase inhibitors from medicinal plants used to treat inflammation," Pharmacological Research, vol. 61, no. 6, pp. 519-524, 2010.

[22] L. Lao, A. Y. Fan, R. X. Zhang et al., "Anti-hyperalgesic and anti-inflammatory effects of the modified Chinese herbal formula Huo Luo Xiao Ling Dan (HLXL) in rats," The American Journal of Chinese Medicine, vol. 34, no. 5, pp. 833844,2006

[23] R. X. Zhang, A. Y. Fan, A. N. Zhou et al., "Extract of the Chinese herbal formula Huo Luo Xiao Ling Dan inhibited adjuvant arthritis in rats," Journal of Ethnopharmacology, vol. 121, no. 3, pp. 366-371, 2009.

[24] Y. H. Yang, R. Rajaiah, D. Y. Lee et al., "Suppression of ongoing experimental arthritis by a Chinese herbal formula (Huo-LuoXiao-Ling Dan) involves changes in antigen-induced immunological and biochemical mediators of inflammation," Evidence-based Complementary and Alternative Medicine, vol. 2011, Article ID 642027, 10 pages, 2011.

[25] Z. Szekanecz, M. M. Halloran, M. V. Volin et al., “Temporal expression of inflammatory cytokines and chemokines in rat adjuvant-induced arthritis," Arthritis \& Rheumatism, vol. 43, no. 6, pp. 1266-1277, 2000.

[26] R. Rajaiah, M. Puttabyatappa, S. K. Polumuri, and K. D. Moudgil, "Interleukin-27 and interferon- $\gamma$ are involved in regulation of autoimmune arthritis," The Journal of Biological Chemistry, vol. 286, no. 4, pp. 2817-2825, 2011.

[27] S. H. Venkatesha, H. Yu, R. Rajaiah, L. Tong, and K. D. Moudgil, "Celastrus-derived celastrol suppresses autoimmune arthritis by modulating antigen-induced cellular and humoral effector responses," The Journal of Biological Chemistry, vol. 286, no. 17, pp. 15138-15146, 2011.

[28] S. A. Komeh-Nkrumah, S. M. Nanjundaiah, R. Rajaiah, H. Yu, and K. D. Moudgil, "Topical dermal application of essential oils attenuates the severity of adjuvant arthritis in lewis rats," Phytotherapy Research, vol. 26, no. 1, pp. 54-59, 2012.

[29] C. M. Brodmerkel, R. Huber, M. Covington et al., "Discovery and pharmacological characterization of a novel rodent-active CCR2 antagonist, INCB3344," The Journal of Immunology, vol. 175, no. 8, pp. 5370-5378, 2005.

[30] Y. Jin, X. Cui, U. P. Singh et al., "Systemic inflammatory load in humans is suppressed by consumption of two formulations of dried, encapsulated juice concentrate," Molecular Nutrition \& Food Research, vol. 54, no. 10, pp. 1506-1514, 2010.

[31] H. Marotte, J. H. Ruth, P. L. Campbell, A. E. Koch, and S. Ahmed, "Green tea extract inhibits chemokine production, but up-regulates chemokine receptor expression, in rheumatoid arthritis synovial fibroblasts and rat adjuvant-induced arthritis," Rheumatology, vol. 49, no. 3, pp. 467-479, 2010.

[32] P. R. Pine, B. Chang, N. Schoettler et al., "Inflammation and bone erosion are suppressed in models of rheumatoid arthritis following treatment with a novel Syk inhibitor," Clinical Immunology, vol. 124, no. 3, pp. 244-257, 2007.

[33] D. Ahrens, A. E. Koch, R. M. Pope, M. Stein-Picarella, and M. J. Niedbala, "Expression of matrix metalloproteinase 9 (96kd gelatinase B) in human rheumatoid arthritis," Arthritis \& Rheumatism, vol. 39, no. 9, pp. 1576-1587, 1996.

[34] S. C. Robinson, K. A. Scott, and F. R. Balkwill, "Chemokine stimulation of monocyte matrix metalloproteinase-9 requires endogenous TNF- $\alpha$," European Journal of Immunology, vol. 32, no. 2, pp. 404-412, 2002.

[35] T. Nanki, K. Nagasaka, K. Hayashida, Y. Saita, and N. Miyasaka, "Chemokines regulate IL-6 and IL-8 production by fibroblast-like synoviocytes from patients with rheumatoid arthritis," The Journal of Immunology, vol. 167, no. 9, pp. 53815385, 2001.

[36] M. J. Ruddy, F. Shen, J. B. Smith, A. Sharma, and S. L. Gaffen, "Interleukin-17 regulates expression of the CXC chemokine LIX/CXCL5 in osteoblasts: implications for inflammation and neutrophil recruitment," Journal of Leukocyte Biology, vol. 76, no. 1, pp. 135-144, 2004. 


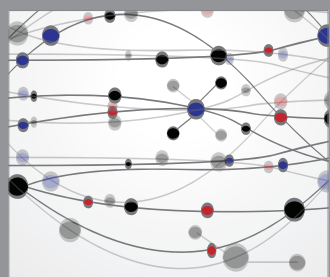

The Scientific World Journal
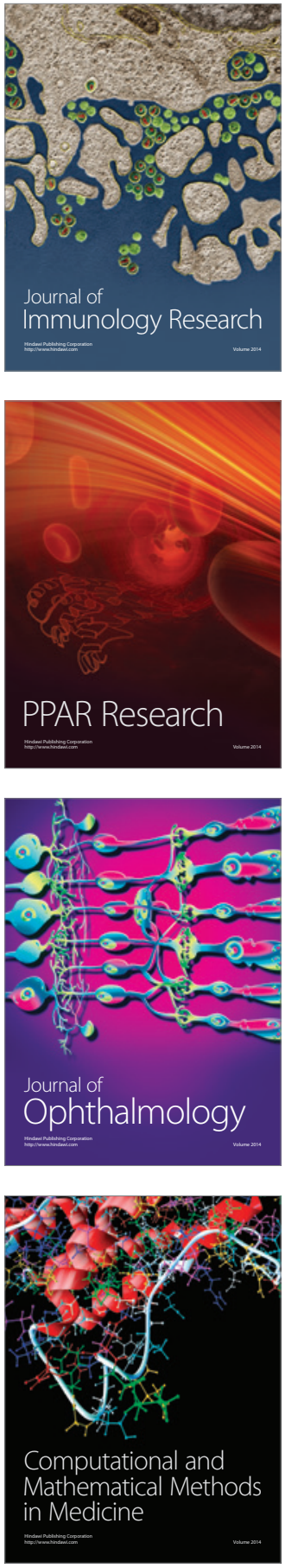

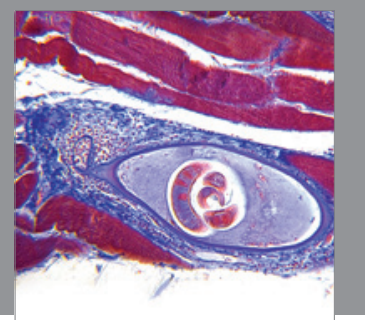

Gastroenterology

Research and Practice
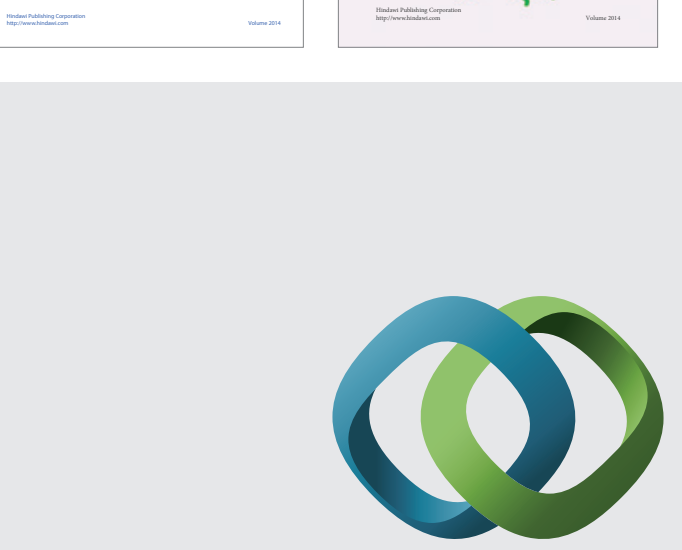

\section{Hindawi}

Submit your manuscripts at

http://www.hindawi.com
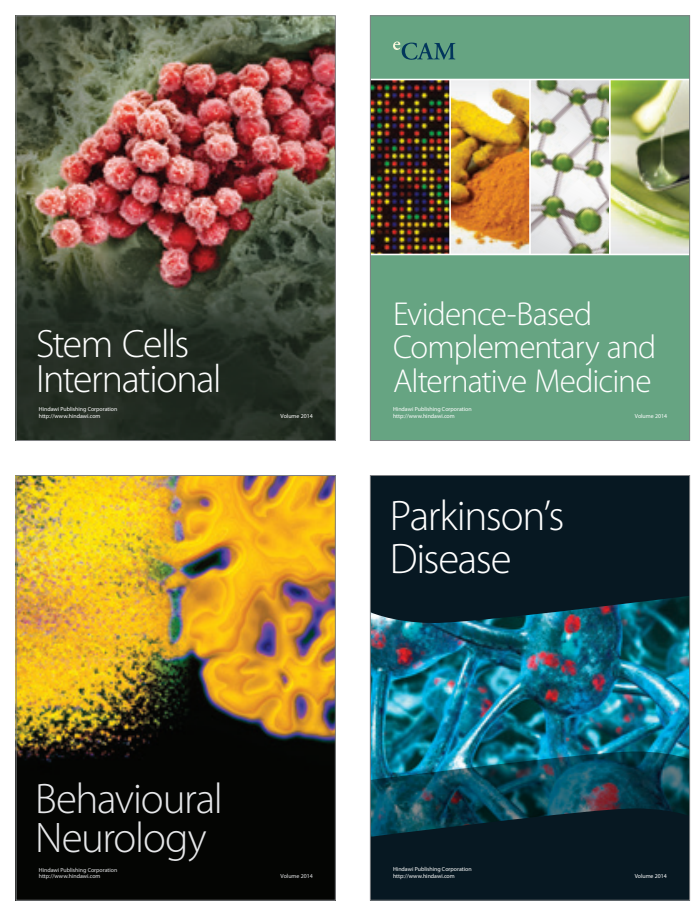

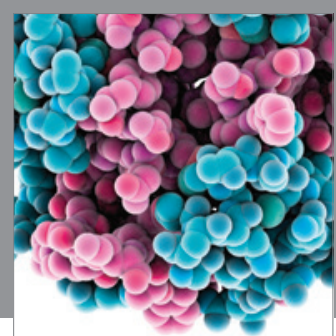

Journal of
Diabetes Research

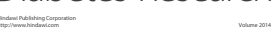

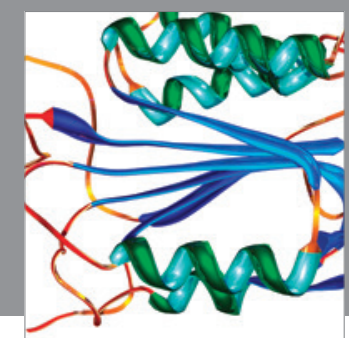

Disease Markers
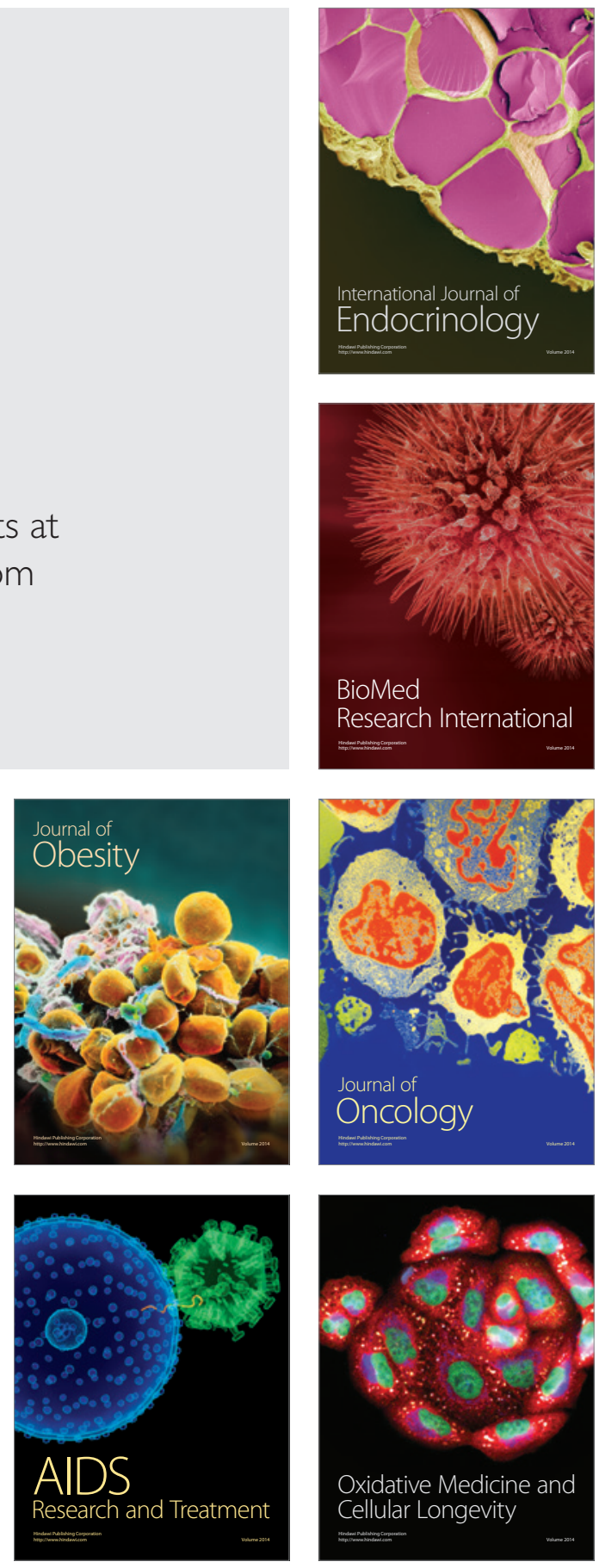